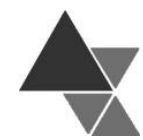

\title{
O desmonte das iniciativas governamentais para a Segurança Alimentar e
} Nutricional: estudo de caso do município de Cuité - Paraíba, entre 2014 e 2019

\author{
Ana Beatriz Macêdo Venâncio dos Santos ${ }^{1}$, Elaine Valdna Oliveira dos Santos ${ }^{2}$, Celena Dantas \\ de Medeiros ${ }^{3}$, Susana Arruda Cordeiro ${ }^{4}$, Ana Bernadete Paiva de Oliveira de Lima ${ }^{5}$, Josiclea \\ Gomes da Silva ${ }^{6}$, Maria dos Aflitos Soares de Oliveira ${ }^{7}$, José Victor de Sousa Lira ${ }^{8}$ e Poliana de \\ Araújo Palmeira ${ }^{9}$
}

O estudo teve o objetivo de analisar a implementação local de iniciativas governamentais federais para alcance da Segurança Alimentar e Nutricional (IG-SAN), a fim de avaliar o desmantelamento de políticas públicas entre 2014 e 2019. Foi realizado um estudo de caso no município de Cuité, Paraíba, por meio do acompanhamento do cenário de IGs-SAN em dois momentos, 2014 e 2019. A coleta de dados contou com mapeamento de IGs-SAN e entrevistas com gestores em 2014 e em 2019. Os resultados revelaram o processo de desmantelamento na implementação local de IGs-SAN marcado tanto pela extinção e inativação de programas federais, como pela fragilização na implementação daquelas iniciativas em curso no município. Em 2014, o cenário de IGs-SAN foi composto por 26 iniciativas. Em 2019, 15 continuavam vigentes e três novas ações foram implementadas. Foram categorizados sinais de desmonte em diferentes frentes: criação de um ambiente político de incerteza, irregularidades/cortes nos recursos financeiros, redução da capacidade do município de executar as ações e solucionar problemas e insuficiência de comunicação entre instâncias federativas. O estudo analisou o processo de desmonte, a partir das relações entre o governo federal e o municipal, revelando que as decisões políticas recentes conduzidas pelo governo federal não promovem o alcance da SAN.

Palavras-chave: Iniciativa governamental; Política pública; Insegurança alimentar; Ação pública local; Direito à alimentação.

\footnotetext{
${ }^{1}$ Doutoranda do Programa de Pós-Graduação em Saúde Coletiva. Universidade Federal do Rio Grande do Norte. Endereço para correspondência: Rua João Pessoa no 111, Cuité - PB, CEP: 58.175-000. Tel. (83)99651-4354. E-mail: abmv.santos@gmail.com. ID ORCID https://orcid.org/0000-0002-1061-6496

2 Docente do Curso de Nutrição da Universidade Federal de Campina Grande, Centro de Educação e Saúde, Núcleo de Pesquisa e Estudos em Nutrição e Saúde Coletiva (Núcleo Penso), Cuité - PB. ID ORCID https://orcid.org/0000-0002-1808-1385

3 Graduanda do Curso de Nutrição da Universidade Federal de Campina Grande, Centro de Educação e Saúde. ID ORCID https://orcid.org/0000-0002-3190-5596

${ }_{4}^{4}$ Graduanda do Curso de Nutrição da Universidade Federal de Campina Grande, Centro de Educação e Saúde. ID ORCID https://orcid.org/0000-0001-9303-4098

5 Graduanda do Curso de Nutrição da Universidade Federal de Campina Grande, Centro de Educação e Saúde. ID ORCID https://orcid.org/0000-0002-3158-9781

${ }^{6}$ Graduanda do Curso de Nutrição da Universidade Federal de Campina Grande, Centro de Educação e Saúde. ID ORCID https://orcid.org/0000-0002-8151-2766

${ }^{7}$ Graduanda do Curso de Nutrição da Universidade Federal de Campina Grande, Centro de Educação e Saúde. ID ORCID https://orcid.org/0000-0003-0646-5346

${ }^{8}$ Graduanda do Curso de Nutrição da Universidade Federal de Campina Grande, Centro de Educação e Saúde. ID ORCID https://orcid.org/0000-0003-4343-6739

9 Docente do Curso de Nutrição da Universidade Federal de Campina Grande, Centro de Educação e Saúde, Núcleo de Pesquisa e Estudos em Nutrição e Saúde Coletiva

(Núcleo Penso), Cuité - PB. ID ORCID https://orcid.org/0000-0002-3503-3414
} 


\section{The dismantling of governmental initiatives for Food Security and Nutrition: a case study in the municipality of Cuité - Paraíba, between 2014 and 2019}

The study aimed to analyze the local implementation of federal government initiatives to achieve Food Security and Nutrition (GI-FSN), to assess the dismantling of public policies between 2014 and 2019. We carried out a case study in the municipality of Cuité, Paraíba, to monitoring of the GIs-FSN scenario in 2014 and 2019. The data collection included mapping of GI-FSN and managers interviews in 2014 and 2019. The results revealed the dismantling process in the local implementation of GI-FSN both due to the extinction and inactivation of federal programs and the fragility in the implementation of those initiatives in place at municipality. In 2014, the GIFSN scenario was composed of 26 initiatives. In 2019, 15 were still in force and three new ones were implemented. We categorized signs of dismantling on different fronts: uncertainty political environment, irregularities / cuts in financial resources, reduced capacity of the municipality to carry out actions and solve problems, and insufficient federative communication. The study analyzed the dismantling process based on the relationship between the National and local governments and revealed that recent political decisions conducted by the federal government do not promote the reach of the FSN.

Keywords: Government initiative; Public policy; Food insecurity; Local public action; Right to food.

Submetido em: 30/10/2020

Aceito em: 12/06/2021

\section{INTRODUÇÃO E OBJETIVOS}

Apesar de décadas de esforços a fome, a má nutrição e a Insegurança Alimentar (IA) permanecem dentre os mais importantes problemas sociais e de saúde pública a serem enfrentados no mundo ${ }^{[1,2]}$. A Organização das Nações Unidas para Alimentação e Agricultura (FAO) apresenta evidências de que, desde 2014, os níveis de insegurança alimentar no mundo têm aumentado gradativamente[ ${ }^{[3]}$. No contexto brasileiro, em 2020, o Instituto Brasileiro de Geografia e Estatística (IBGE) confirmou a maior exposição da população brasileira à IA, revelando que entre 2013 e 20172018 o percentual de famílias IA aumentou de 22,6 $\%$ para $36,7 \%[4]$.

A a violação do direito de todas as pessoas ao acesso a alimentos suficientes, adequados e seguros ${ }^{[5]}$ demanda interesse político e público na implementação de políticas para a garantia da Segurança Alimentar e Nutricional (SAN). Desta forma, estas últimas visam promover crescimento econômico, desenvolvimento rural e proteção social na construção de sistemas alimentares saudáveis, inclusivos e sustentáveis ${ }^{[6-8]}$.
O governo brasileiro foi pioneiro no desenvolvimento de políticas públicas de SAN e, entre 2003 e 2014, implementou um conjunto coordenado de políticas e programas cujos efeitos foram associados à redução consistente nas taxas de IA no país ${ }^{[9-11]}$. Estudo realizado em um município da região Nordeste do Brasil revelou que, neste período, o governo nacional implementou intervenções coordenadas que incentivaram a governança da SAN na esfera municipal, o que aumentou a capacidade político-administrativa local e criou uma conjuntura que promoveu o acesso a programas em diferentes áreas de governo, contribuindo para a superação da IA[12].

No entanto, pesquisadores e a comunidade em geral têm alertado e acompanhado o desmonte progressivo da política nacional de SAN, instituída em 2006 por meio da Lei Orgânica de SAN[13]. Desmonte é um termo usado para identificar indícios de desarticulações, frágeis ou profundas, de políticas públicas ou programas governamentais, caracterizando-se por uma fragilização da garantia de direitos das pessoas ${ }^{[14]}$. Nessa perspectiva, o desmantelamento pode se expressar pela intenção e/ou decisão política por encerrar o funcionamento ou reduzir gradualmente 
em termos de poder ou propósito programas ou arranjos políticos.

A luz deste conceito, desde 2016, o Brasil enfrenta uma forte crise econômica agravada pela instabilidade política, que resultou na implementação de medidas de austeridade fiscal[15,16]. Segundo Vasconcelos e Machado[17] durante o governo de Michel Temer (2016-2018), houve intensificação de tais medidas com cortes orçamentários que fragilizaram políticas sociais e de SAN no país ${ }^{[16]}$. Na mesma direção, em 2019, o Governo Bolsonaro extinguiu o Conselho Nacional de Segurança Alimentar e Nutricional (CONSEA), espaço de diálogo e controle social entre representantes da sociedade civil e do Estado[18].

Desta forma, a redução progressiva do investimento federal em políticas de saúde pública, educação e proteção social[19,20] implica em fragilização da implementação local de sistemas universais e programas governamentais de assistência à população, dado que a cooperação intergovernamental é um arranjo próprio do federalismo do Estado Brasileiro ${ }^{[21]}$. Assim, especialmente para os municípios com maior dependência financeira e político-administrativa do governo federal, como os municípios de pequeno porte, este cenário de redução do Estado engajado na garantia de direitos sociais pode ter consequências devastadoras para a construção de uma governança local para promoção da SAN da população ${ }^{[12]}$.

Portanto, diante da gravidade do problema, este estudo visa analisar os impactos do desmonte de políticas públicas para o alcance da $\mathrm{SAN}$, considerando o quadro nacional para focalizar o cenário local de implementação de dispositivos governamentais federais relacionados com a SAN.

\section{MATERIAL E MÉTODOS}

Este estudo se refere à análise de política pública na perspectiva do processo de implementação local[22] de iniciativas propostas pelo Governo Federal para o alcance da a SAN (IGSAN). Para tanto, foi realizado estudo de caso no município de Cuité com o objetivo de identificar e caracterizar iniciativas existentes, além de avaliar o grau de desmonte de dispositivos públicos, comparando seus processos de implementação em dois momentos (2014 e 2019).

O município está localizado no semiárido nordestino, região conhecida por períodos de seca frequentes, altas temperaturas e economia ligada à pecuária e agricultura [23]. O município de Cuité, onde foi realizada a pesquisa, está localizado a 235 $\mathrm{km}$ da capital do estado da Paraíba (Brasil), ocupa extenso território de aproximadamente $740 \mathrm{~km}^{2} \mathrm{e}$ possui cerca de 20 mil habitantes. Cuité é classificado como um município de baixo índice de desenvolvimento humano $(0.591)^{[24]}$ e pequeno porte, características de aproximadamente $70 \%$ dos municípios brasileiros. A vulnerabilidade social da região do semiárido nordestino expressa notadamente risco para a IA. Ou seja, o município apresenta características que demandam a implementação de políticas na área de SAN, o que constitui tema pertinente para uma investigação aprofundada com um estudo de caso.

\section{Coleta de dados}

O estudo sobre as IGs-SAN existentes no município de Cuité se iniciou em 2011, quando foi realizado o primeiro diagnóstico municipal sobre as políticas locais de SAN. Em 2014, após a implementação do pacote de ações da estratégia "Brasil sem Miséria", foi dada continuidade à pesquisa com a realização de nova coleta de dados. Como resultado, foram identificadas 33 iniciativas vigentes no município em 2014, sendo 26 de origem federal, ou seja, desenhadas pelo governo federal para implementação descentralizada no município[12]. Em 2019, considerando o início de uma nova gestão federal, realizou-se nova pesquisa de campo para acompanhar a situação de implementação das iniciativas mapeadas em 2014 e identificar novas ações propostas pelo novo governo federal (2019-atual).

Neste estudo, foram considerados os dados coletados em 2014 e 2019 em duas etapas: (1) mapeamento de IGs-SAN e (2) entrevistas com gestores e responsáveis técnicos. Outras informações sobre a coleta de dados realizada em 2014 podem ser consultadas em Palmeira e Mattos ${ }^{[12]}$. A seguir serão descritas as etapas de coleta e análise de dados desenvolvidas no ano de 2019. 


\section{Mapeamento de iniciativas governamentais}

Para este estudo, IG-SAN foi definida como qualquer programa, equipamento público ou arranjo institucional proposto pelo governo federal que atue para (i) promoção de sistemas alimentares sustentáveis e (ii) superação das consequências indesejáveis dos sistemas alimentares vigentes, como a IA, a fome e o comprometimento da saúde.

Com base neste contexto, inicialmente realizou-se o mapeamento das IGs-SAN informadas pelo governo federal como vigentes, em acordo com as informações disponíveis nos sites governamentais. Para orientar as visitas técnicas de mapeamento, foi elaborada uma lista de IGs-SAN por secretaria municipal, na qual foram incluídas aquelas identificadas nos sites, junto a outras que compuseram o cenário de IGs-SAN implementadas no município estudado em 2014 (26 iniciativas listadas no Quadro 1). A partir desta listagem, foram realizadas visitas técnicas nas secretarias municipais de saúde, educação, agricultura e assistência social para identificar as iniciativas que permaneceram em vigor no município em 2019. Além das IGs-SAN citadas, foi questionada durante a visita a existência de outras iniciativas no município.

\section{Entrevistas}

Foram selecionadas para realização de entrevista com os gestores as iniciativas implementadas no município após a coleta de dados no ano de 2014 e aquelas que ocupam posição estratégica para a política local de SAN. Assim, dentre as 23 IGs-SAN mapeadas, 15 foram selecionadas para realização de entrevista com gestores: (1) Secretaria de Educação: Mais Educação, Programa Nacional de Alimentação Escolar, Brasil Alfabetizado; (2) Secretaria de Assistência Social: Bolsa Família, Centro de Referência da Assistência Social, Criança Feliz; (3) Secretaria de Saúde: Programa Saúde na Escola, Suplementação de Vitamina A, Suplementação de Ferro, Crescer Saudável, NutriSUS, Sistema Nacional de Vigilância Alimentar e Nutricional, Mais médicos pelo Brasil e Núcleo de Apoio à Saúde da Família; e (4) Secretaria de Agricultura: Garantia Safra.
Para as entrevistas, foi elaborado um roteiro estruturado a partir de três objetivos: (i) caracterizar e mapear as ações realizadas, (ii) caracterizar o processo de implementação local da iniciativa (iii) identificar mudanças na forma do governo federal propor a implementação e a execução do programa no município no ano de 2019 e possíveis desdobramentos.

As entrevistas foram áudio-gravadas e realizadas nas secretarias municipais, por entrevistadores previamente treinados entre novembro e dezembro de 2019. Dentre as 15 iniciativas selecionadas para entrevista, ao final do trabalho de campo, treze foram efetivamente realizadas. Não foi possível realizar a entrevista sobre Médicos pelo Brasil e Núcleo de apoio à saúde da família devido a dificuldades de agendamento com o gestor.

\section{Análise de dados}

Para análise de dados, todas as entrevistas foram transcritas na íntegra, analisadas e codificadas no software Atlas.ti 7.5.4, Berlin[25], tendo sido codificadas por 4 autores do artigo. Os códigos, temas e categorias foram revisados e validados por outros dois autores. Quando necessário, de forma complementar, realizou-se pesquisa documental, em sites oficiais do governo federal e municipal, sobre as iniciativas estudadas visando subsidiar a entrevista com os gestores e a análise de dados. Antes desta última, parte dos autores realizou leituras exploratórias das entrevistas com o objetivo de planejar seu trabalho analítico. Baseado na leitura prévia, o conteúdo das entrevistas foi analisado em duas etapas.

A primeira etapa teve como objetivo comparar os cenários de IGs-SAN implementadas no município nos anos de 2014 e 2019. Com base nos dados coletados, as 26 IGs-SAN vigentes no município em 2014 e as novas identificadas graças às informações obtidas em 2019 foram classificadas de acordo com o ano e a situação de implementação no município em três categorias: (i) Vigente: iniciativa cujos gestores municipais informaram que as ações ou benefícios estão sendo ofertados para a comunidade; (ii) Inativa: iniciativa não foi citada por gestores como oficialmente extinta, no entanto, não estavam sendo ofertadas ações ou benefícios há 
pelo menos um ano ou as atividades foram suspensas em 2019; (iii) Extinta: iniciativa citada no mapeamento como não mais existente no município.

A segunda etapa visou categorizar e analisar sinais de desmonte dentre as iniciativas vigentes no município. A análise realizada está fundamentada na teoria proposta por Ball[26] que pressupõe que as políticas estão em constante movimento, delimitando uma trajetória incerta em contextos distintos, de forma que a apreciação da política a partir do contexto pode revelar como se move, se transforma e se remodela em diferentes cenários. Desta forma, como proposto por Mattos e Baptista ${ }^{[22]}$, buscou-se a partir das práticas sociais dos gestores reconhecer e compreender sinais de desmonte a partir de foco sobre o contexto no qual a IG-SAN estava inserida.

Nesta etapa, foram analisadas entrevistas sobre 12 IGs-SAN listadas no Quadro 2. Não foram incluídas na análise as iniciativas Saúde de Ferro, pois o gestor relatou não ter informações sobre o programa dado que estava inativo, e Mais Educação, pois o arquivo da entrevista foi corrompido, impossibilitando a obtenção de suas informações para categorização. Durante a entrevista com o gestor do Centro de Referência da Assistência Social foi abordado o programa Benefício de Prestação Continuada, por uma demanda do entrevistado. Assim, tal iniciativa foi incluída na análise.

Ao final da análise de dados, foram categorizados oito sinais de desmonte, distribuídos em 4 eixos temáticos definidos pelos autores: (i) Recursos financeiros, que inclui sinais de desmonte relacionados com irregularidade, instabilidade ou corte nos repasses de recursos financeiros $\mathrm{e}$ humanos; (ii) Comunicação e acesso à informação, categoria que engloba desmonte relacionado com a ausência efetiva na comunicação ou restrição de acesso a informações; (iii) Oferta das ações e benefícios, que inclui fragilização da oferta dos serviços no tocante à cobertura do público-alvo e às oportunidades de acesso pela população e; (iv) Continuidade, que descreve a insegurança com relação à permanência da iniciativa em curto e longo prazo.
Os oito sinais de desmonte categorizados foram sistematizados no Quadro 2, com o objetivo de visualizar sua distribuição no conjunto das iniciativas estudadas e de viabilizar a comparação e a análise da concentração de problemas em secretarias municipais e em uma mesma IG-SAN.

Os resultados serão apresentados em duas seções: (1) Implementação local de iniciativas governamentais relacionadas com SAN, na qual será apresentada a situação de implementação comparando o cenário de IGs-SAN existente em 2014 com aquele identificado em 2019, e (2) Sinais de desmantelamento, onde serão descritas as categorias de desmontes observadas dentre as iniciativas vigentes no município em 2019.

O projeto de pesquisa foi aprovado pelo Comitê de Ética em Pesquisa do Hospital Universitário Alcides Carneiros da UFCG em 2014 (CAAE: 30919314.6.0000.5182). Em 2019, foi aprovada a extensão do projeto para realização de nova coleta de dados.

\section{RESULTADOS E DISCUSSÃO}

\section{Implementação local de iniciativas governamentais relacionadas com SAN}

Em 2014, existiam 26 IGs-SAN implementadas no município focalizado. Dentre estas iniciativas, em 2019, 15 foram classificadas como vigentes, 10 estavam com atividades interrompidas, das quais cinco inativas e outras cinco haviam sido extintas. Uma das ações era de responsabilidade do governo estadual e foi excluída da análise (Assistência Técnica e Extensão Rural) (Quadro 1). Em acordo com a linha do tempo exposta no Quadro 1, houve maior proposição de IGs-SAN pelo governo federal entre os anos de 2009 e 2014 (13 IG-SAN). Apenas três iniciativas foram implementadas a partir de 2014: Criança Feliz (2018), Crescer Saudável (2019) e Mais Médicos pelo Brasil (2019).

Em 2019, o cenário no município foi composto por 18 IGs-SAN implementadas, com ações e benefícios ofertados para a população. Deste conjunto, nove IGs-SAN estavam alocadas na secretaria municipal de Saúde, cinco na 
Assistência Social, duas na Agricultura e mais duas na Educação.

As inciativas classificadas como inativas ou extintas estavam distribuídas em diferentes setores governamentais: Agricultura (Programa Uma Terra duas águas, Distribuição de Sementes e
Programa Um milhão de Cisternas), Saúde (Rede Cegonha, Mais Médicos, Programa Saúde de Ferro) Educação (Ensino médio inovador, Brasil Alfabetizado), Assistência Social (Programa Nacional de Acesso ao Ensino Técnico e Emprego, Serviço de Convivência e Fortalecimento de Vínculos).

Quadro 1. Ano e situação de implementação de iniciativas governamentais de origem federal relacionadas com segurança alimentar e nutricional, com base em mapeamentos realizados em 2014 e 2019, Cuité, Paraíba, Brasil

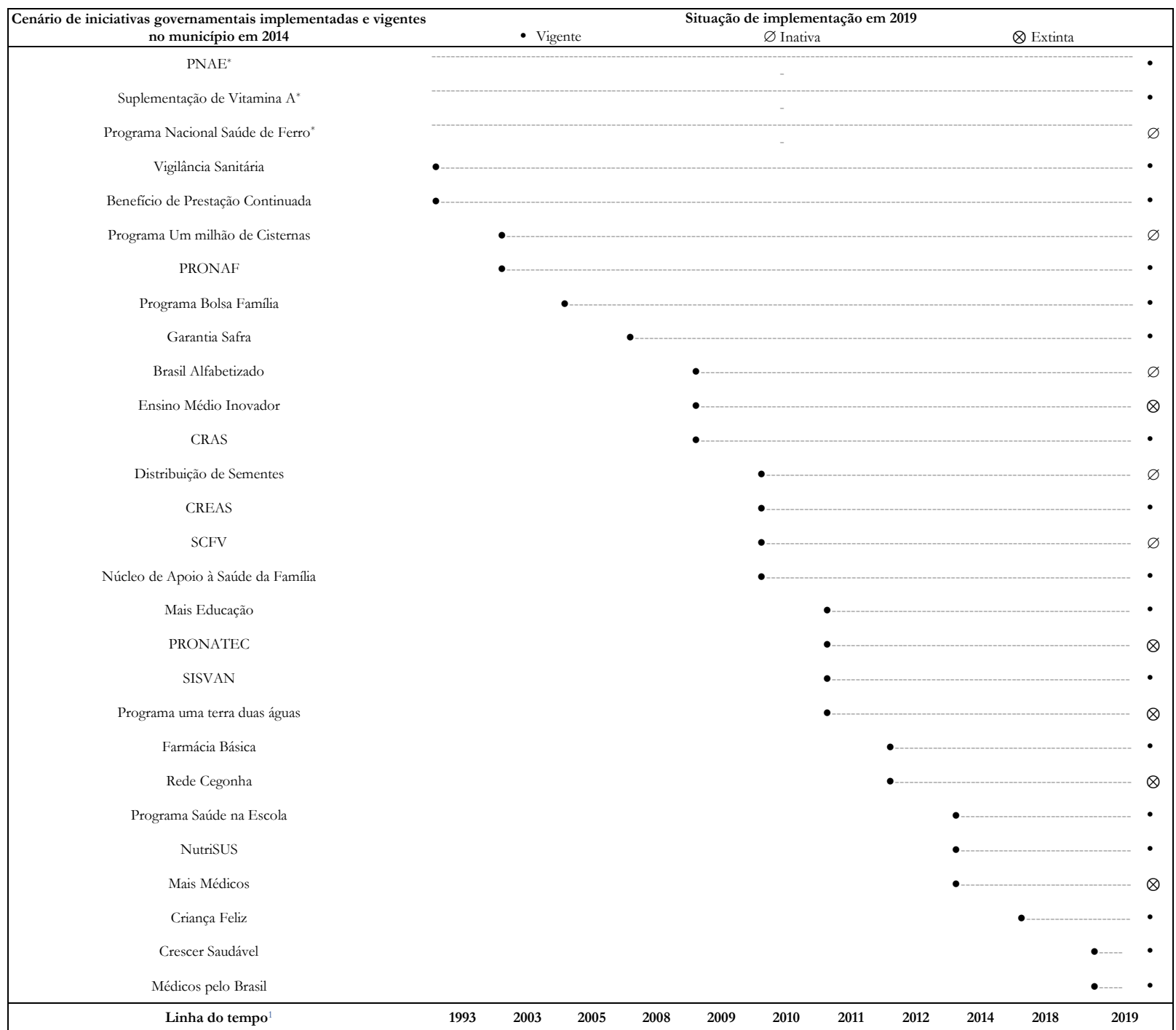

Nota: PNAE: Programa Nacional de Alimentação Escolar; PRONAF: Programa Nacional de Fortalecimento da Agricultura Familiar; CRAS: Centro de Referência da Assistência Social; CREAS: Centro de Referência Especializado de Assistência Social; SCFV: Serviço de Convivência e Fortalecimento de Vínculos; PRONATEC: Programa Nacional de Acesso ao Ensino Técnico e Emprego; SISVAN: Sistema de Vigilância Alimentar e Nutricional.

1 Ano de implementação das iniciativas marcadas na linha do tempo com o círculo preto. Governo Itamar Franco (1992-1995), Governo Luiz Inácio Lula da Silva (2003-2010), Governo Dilma Rousseff (2011-2016), Governo Michel Temer (2016-2018), Governo Jair Bolsonaro (início em 2019).

*Ano de implementação da iniciativa governamental não foi identificado durante a coleta de dados 
Dentre aquelas IGs-SAN classificadas como inativas, verificou-se o abandono do repasse de suplementos nutricionais e de sementes do governo federal para o município, referentes aos programas Saúde de Ferro e Distribuição de Sementes, respectivamente. Verificou-se, também, a ausência de repasse de recursos financeiros ao governo local, inviabilizando a oferta dos serviços, no caso das seguintes iniciativas: Programa Um milhão de Cisternas, Brasil Alfabetizado e Serviço de Convivência e Fortalecimento de Vínculos.

\section{Sinais de desmonte na implementação de iniciativas governamentais relacionadas com SAN}

O Quadro 2 apresenta oito sinais de desmonte codificados nas entrevistas com os gestores agrupados em quatro eixos temáticos. No Eixo Recursos Financeiros, estão descritas duas situações de irregularidade ou instabilidade no repasse de recursos ou do material necessário para execução das ações (Sinal 1) e corte no recurso destinado à oferta do benefício e ao pagamento de recursos humanos (Sinal 2), os quais foram observados em cinco e quatro iniciativas, respectivamente. No Eixo Comunicação e acesso à informação, também foram constatados os seguintes problemas: ausência de comunicação efetiva e transparente entre governo federal e municipal para dar suporte na gestão das ações (Sinal 3) e restrição do acesso aos sistemas de informação (Sinal 4). Ausência de comunicação efetiva e transparência foi o sinal de desmonte mais observado dentre as iniciativas estudadas (9 iniciativas) e a restrição no acesso aos sistemas de informação foi citada por cinco gestores.

No Eixo Oferta das ações e benefícios, foram verificadas três situações relacionadas com a diminuição da cobertura populacional (Sinal 5), a redução de oportunidades no acesso ao conjunto de benefícios ofertados pela iniciativa (Sinal 6) e o não reconhecimento das vulnerabilidades climáticas locais como justificativa para não ofertar benefícios (Sinal 7), os quais foram observados em três, cinco e uma IG-SAN, respectivamente. No quarto eixo denominado de Continuidade, foi identificado o aumento da insegurança quanto ao prosseguimento da iniciativa em função de mudanças recentes na política conduzida no nível federal (Sinal 8), citado por oito gestores entrevistados.

A análise dos resultados do Quadro 2 a partir das IGs-SAN estudadas, permite observar que todas as iniciativas apresentaram pelo menos um sinal de desmonte, com muitas sendo fortemente fragilizadas. O Centro de Referência da Assistência Social, Programa Bolsa Família e Garantia Safra estão entre as iniciativas que apresentaram uma concentração maior de sinais de desmonte (5 ou mais). Observou-se entre três e cinco sinais nas IGs-SAN Benefício de Prestação Continuada, Brasil Alfabetizado, Programa Nacional de Alimentação Escolar, Suplementação de Vitamina A e NutriSus. Menos de três sinais foram constatados nas iniciativas Criança Feliz, Programa Saúde na Escola, Sistema de Vigilância Alimentar e Nutricional e Crescer Saudável.

Este estudo revelou, portanto, um desmantelamento em curso na implementação local de diferentes IGs-SAN conduzido pelo governo federal, cujas características estão marcadas tanto pela extinção e inativação de programas federais específicos, como pela fragilização na implementação daquelas iniciativas ainda vigentes no município estudado em 2019. O desmonte foi observado em diferentes frentes: criação de um ambiente político de incerteza, irregularidades e cortes nos recursos financeiros, redução da capacidade do município de executar as ações e solucionar problemas, e insuficiência de comunicação entre instâncias federativas (federalmunicipal). 
Quadro 2. Sinais de desmonte observados na implementação local de iniciativas governamentais relacionadas com segurança alimentar e nutricional propostas pelo governo federal e descentralizadas ao município, Cuité, Paraíba, Brasil, 2019

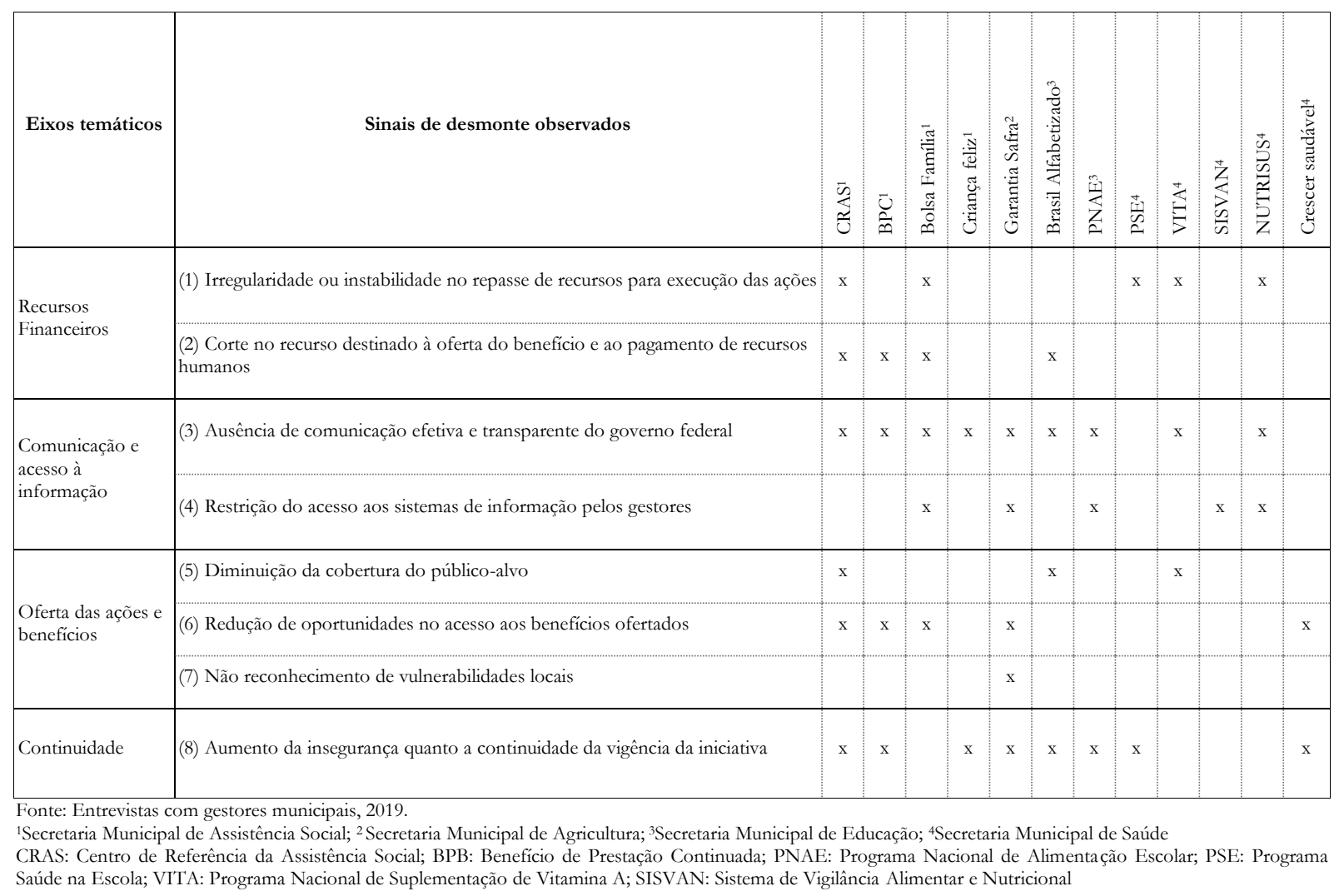

Segundo Marquese Roberto[14], o desmonte das políticas públicas brasileiras se configura na presença de estratégias que visam fragilizar as políticas públicas por meio de princípios neoliberais e modificações legislativas que podem resultar em violação de direitos sociais básicos. Esses retrocessos ocorrem em um contexto de déficit no crescimento econômico e de alto índice de desemprego, potencializando os impactos negativos sobre o bem-estar da população, o que é reforçado por um Estado reduzido, pouco articulado e, portanto, ineficiente para o enfrentamento de problemas sociais complexos como a IA.

Neste contexto de desmonte da ação do Estado, estudos apontam impactos desfavoráveis da política de austeridade fiscal na saúde e proteção social da população[27], como observado para os sistemas únicos de saúde da Espanha ${ }^{[28]}$ e do Reino Unido ${ }^{29]}$, especialmente entre a população com maior vulnerabilidade. No Brasil, pesquisadores avaliaram o investimento do Governo Federal em 19 programas governamentais relacionados com os Objetivos de Desenvolvimento Sustentável e observaram que em apenas quatro programas houve aumento de orçamento entre 2014 e 2017. Nos programas relacionados com SAN verificou-se uma redução no orçamento de $84,6 \%$ [20].

Esse controle dos gastos públicos no Brasil com cortes orçamentários em diferentes campos de políticas sociais, como SUS, SUAS, apoio à agricultura familiar, educação superior, previdência e outros ${ }^{[30]}$, pode vir a ter um expressivo impacto principalmente entre os grupos 
populacionais mais vulneráveis, como a população de baixa renda e rural.

Desde a gestão do governo federal no período 2016-2018, nota-se que, progressivamente, pautas relacionadas à SAN, bem como à garantia do DHAA, perderam visibilidade e passaram a constituir políticas de importância secundária. A extinção do CONSEA em 2019 constitui o principal marco relacionado com o desmantelamento das ações voltadas à SAN [15,31]. Durante sua existência, este conselho foi componentes fundamental do Sistema Nacional de Segurança Alimentar e Nutricional, cujo objetivo era formular e implementar políticas e planos de SAN, com perspectiva de descentralização aos municípios ${ }^{[13]}$, assumindo um importante papel na defesa e indução de pautas e políticas relacionadas com a SAN da população ${ }^{[12]}$. A extinção do CONSEA representa, portanto, um retrocesso social, pois visa calar um espaço de vigilância na qual a representação da sociedade civil possui vOZ ativa nos processos decisórios relacionados ao DHAA no Brasil[32].

Dessa forma, a redução dos investimentos públicos federais e a ausência de uma coordenação nacional na construção da política descentralizada de SAN têm impacto no cenário de iniciativas e na capacidade dos governos locais de combater a IA e superar as vulnerabilidades sociais, como observado no município estudado. Como ressaltam Silva e Panelli-Martins ${ }^{[3]}$, quando as esferas federal e estadual apresentam instâncias do Sistema de Segurança Alimentar e Nutricional (SISAN) robustas, a política local de SAN pode ser beneficiada, na medida em que recebe suporte para enfrentamento dos desafios correntes à sua implementação.

Nesta perspectiva de desengajamento do Estado, observou-se que após 2014 foram implementados no município apenas três programas (Médicos pelo Brasil, Criança Feliz e Crescer Saudável). Ou seja, na medida em que IGs-SAN foram inativadas ou extintas entre 2014 e 2019, não houve a reposição dessas ações por meio da criação e implementação de novas iniciativas, exceto para o programa Mais Médicos. Em 2019, o provimento de médicos na Atenção Básica passou a ser realizado por meio do programa Médicos pelo Brasil, cujo desenho foi alvo de críticas por reduzir o escopo de ações da Atenção Primária à Saúde e não abarcar estratégias de formação e fixação de médicos no SUS[34,35].

Apesar da constatação geral de desmonte, foi possível observar singularidades do processo de desmantelamento de iniciativas em diferentes secretarias municipais.

No setor de Assistência Social, a inativação do Serviço de Convivência e Fortalecimento de Vínculos em 2019, juntamente com os relatos de cortes, irregularidades nos aportes de recursos financeiros, aumento da burocratização do acesso aos beneficiários e incerteza quanto à continuidade de programas estratégicos para a referida política municipal, evidenciam o desmantelamento em curso. Tais fragilidades reduzem a capacidade local de gestão, pois são limites para as atividades de planejamento e oferta regular de serviços públicos.

Cabe destacar também o contexto de incerteza quanto à continuidade dos programas considerados. Observou-se a inquietude do gestor local ao mencionar espontaneamente informações sobre o Benefício de Prestação Continuada em entrevista sobre o Centro de Referência da Assistência Social (CRAS), enfatizando a obscuridade do governo federal na orientação dos municípios quanto às mudanças na gestão das iniciativas. Como exemplo, citou a retirada desse centro CRAS como referência para o cidadão que busca o Benefício de Prestação Continuada, agora devendo ter contato direto com o Instituto Nacional de Seguridade Social.

Para este setor, os resultados apontaram ainda que em apenas uma iniciativa (Criança Feliz) não foi observado redução de recursos financeiros e oferta de ações e benefícios, caracterizando um avanço frágil, devido ao investimento isolado e desproporcional em uma iniciativa, enquanto há o desmonte em curso nas demais iniciativas que a sustentam no âmbito da Política Nacional de Assistência Social[14]. Este cenário alinha-se àquele nacional apresentado por Teixeira e Silva[36] caracterizado pela redução dos investimentos orçamentários e de serviços da política de Assistência Social, devido ao aumento da burocracia 
e da ultra seletividade de beneficiários. Segundo as autoras, a redução do papel do Estado enquanto responsável por garantir direitos básicos a todos é o plano de fundo do desmantelamento, por meio da difusão de um discurso de "autonomia da família" ou familismo, situação em que o governo transfere a responsabilidade de superação das vulnerabilidades para as próprias famílias.

No âmbito das iniciativas situadas no âmbito da Agricultura, o desmantelamento se revela pelo enxugamento da pasta setorial, principalmente devido à falta de repasse de recursos para os programas Distribuição de Sementes e Um milhão de cisternas em 2019, além da transferência da gestão municipal do Programa Nacional de Fortalecimento da Agricultura Familiar para os órgãos diretos de financiamento. Tal ausência ou precarização de incentivos do Estado reflete na SAN das comunidades, principalmente considerando as adversidades climáticas do semiárido.

De acordo com Rocha[ ${ }^{[3]}$, a ausência e extinção de programas estruturantes faz com que problemas periódicos, como é o caso da seca no semiárido, sejam tratados de forma emergencial e pouco resolutiva. $\mathrm{O}$ investimento em políticas públicas agrárias de grande alcance social visa resgatar a cultura alimentar e minimizar danos sociais, principalmente em meio a uma política agrícola de tecnologias voltadas ao agronegócio, como o caso brasileiro.

Também merece destaque no setor Agricultura o programa Garantia Safra. Nos últimos três anos, o gestor municipal e os agricultores decidiram realizar a adesão ao programa, efetivaram a contrapartida financeira e comprovaram a perda de safra devido à seca. No entanto, o governo federal alegou que não houve estiagem no município para não cumprir seus compromissos. $\mathrm{O}$ contexto de implementação em 2019 deste programa revelou o aumento da burocracia, a inexistência de reconhecimento das vulnerabilidades climáticas do território pelo governo federal, a ausência de comunicação efetiva da esfera federal com o gestor local e a restrição do acesso aos sistemas de informação, culminando em ambiente de incerteza e dificuldades para gerenciamento do programa no município.
No setor Educação, também foi observado o enxugamento de iniciativas. No âmbito do Programa Nacional de Alimentação Escolar, cujas ações são estratégicas tanto para o incentivo à agricultura familiar como para a garantia de acesso aos alimentos para crianças matriculadas da rede pública, dificuldades na comunicação com o governo federal têm prejudicado a resolução de problemas ou a compreensão das mudanças de sua gestão.

O setor Saúde concentrou o maior número de iniciativas vigentes no município. No entanto, entre 2014 e 2019, houve mesmo assim desarticulação das iniciativas Rede Cegonha e Saúde de Ferro, além de redução na cobertura do públicoalvo do Programa Nacional de Suplementação da Vitamina A e irregularidade ou corte no recebimento de recursos em três das cinco iniciativas estudadas no setor. Estes resultados sinalizam para evidentes desmontes em programas relativos à Atenção Primária à Saúde, que compõem um conjunto de ações estratégias para a implementação da Política Nacional de Alimentação e Nutrição nos territórios. Tal constatação corrobora com o processo de desmantelamento do SUS identificado por diferentes pesquisadores ${ }^{[19,38]}$.

A redução dos gastos públicos guiada por medidas de austeridade fiscal e crise econômica pode produzir no Brasil efeitos mais graves do que aqueles observados em países desenvolvidos, considerando o profundo grau de desigualdade social e subfinanciamento para a área da saúde. Esta última está muito ameaçada de sucateamento, o que coloca em risco seu caráter universal[39]. Os efeitos não são imediatos, mas sim ocorrem em longo prazo, dificultando o fortalecimento do SUS como sistema público e universal[40].

Considerando as particularidades de cada setor na perspectiva da SAN, embora a existência e a gravidade do desmonte seja generalizada, a análise aponta para um processo de desmantelamento mais acelerado nos setores de Assistência Social e Agricultura, quando comparado com Saúde e Educação.

No entanto, importa ressaltar que qualquer sinal de desmonte das políticas públicas que visam a garantia da SAN impacta negativamente 
as condições de vida da população. Os impactos diretos na produção e no acesso aos alimentos são iminentes e conduzem a população à extrema

\section{CONCLUSÃO}

Este estudo investigou o cenário de desmantelamento de políticas públicas e iniciativas relacionadas com a SAN em um município de pequeno porte no semiárido nordestino. O foco deste estudo foi analisar o processo de desmonte da implementação de iniciativas governamentais, considerando as relações entre os governos federal e municipal. As mudanças, desmontes e retrocessos observados nesse município também estão em curso em outros territórios. Assim, é recomendável a realização de novos estudos para análise do grau de desmantelamento de políticas públicas em outras realidades brasileiras. Observou-se enxugamento de programas e sinais de desmantelamento foram encontradas na implementação local de iniciativas nas quatro secretarias municipais contempladas no estudo. A fragilização da ação do Estado é mais acelerada no setor da Assistência Social e Agricultura.

Este cenário de desmantelamento das políticas públicas leva ao questionamento das tomadas de decisões que supervalorizam questões ideológicas e econômicas, priorizando austeridade fiscal pautada em lógica neoliberal, em detrimento da garantia de direitos da população. Assim, as decisões políticas recentes não asseguram a SAN, desconsiderando, portanto, a proteção da população e a garantia de direitos aos brasileiros.

\section{AGRADECIMENTOS}

Os autores agradecem, com muito carinho, ao professor Ruben Mattos (in memorian), que com seus ensinamentos inspirou e vibrou com a realização do trabalho. Os autores agradecem, também, aos gestores/profissionais que gentilmente aceitaram colaborar com essa pesquisa. vulnerabilidade com aumento da IA grave, o que, como destacado por Ribeiro-Silva, Pereira ${ }^{[41]}$, pode ser fatal.

\section{REFERÊNCIAS}

[1] Roncarolo F, Potvin L. Food insecurity as a symptom of a social disease: Analyzing a social problem from a medical perspective. Canadian Family Physician. 2016;62(4):291-292.

[2] Loopstra R, Reeves A, Stuckler D. Rising food insecurity in Europe. The Lancet. 2015;385 (9982):2041.

[3] FAO, IFAD, UNICEF, WFP, WHO. The State of Food Security and Nutrition in the World 2020. Transforming food systems for affordable healthy diets. In: FAO, editor. Rome: FAO; 2020.

[4] IBGE. Pesquisa de orçamentos familiares 2017-2018: análise da segurança alimentar no Brasil. Rio de Janeiro: IBGE - Coordenação de Trabalho e Rendimento; 2020.

[5] FAO. Trade Reforms and Food Security: conceptualizing the linkages [Internet]. 2003 [acesso em 2020 jun 15]. Roma: FAO; 2003. Disponível em: http://www.fao.org/docrep/005/y4671e/y4671e00.htm.

[6] Rai RK, Kumar S, Sekher M, Pritchard B, Rammohan A. A life-cycle approach to food and nutrition security in India. Public Health Nutr. 2015;18(5):944-949.

[7] Pirkle CM, Poliquin H, Sia D, Kouakou KJ, Sagna T. Re-envisioning global agricultural trade: time for a paradigm shift to ensure food security and population health in low-income countries. Glob Health Promot. 2015;22(1):60-63.

[8] Friel S, Ford L. Systems, food security and human health. Food sec. 2015;7:437-451.

[9] Silva JG, Del-Grossi ME, França CG. Fome Zero (Zero Hunger) Program: The Brazilian experience. Brasília: MDA; 2010.

[10] Falcão T, Costa PV. Brazil without Extreme Poverty: New Perspectives for Brazilian Social Protection. One Pager 301, International Policy Centre for Inclusive Growth; 2015. 
[11] Palmeira PA, Salles-Costa R, Pérez-Escamilla R. Effects of family income and conditional cash transfers on household food insecurity: evidence from a longitudinal study in Northeast Brazil. Public Health Nutrition. 2020;1-12.

[12] Palmeira P, Mattos R, Salles-Costa R. Food security governance promoted by national government at the local level: a case study in Brazil. Food Security. 2020;12(3):591-606.

[13] Brasil. Lei no 11.346, de 15 de setembro de 2006. Cria o Sistema Nacional de Segurança Alimentar e Nutricional - SISAN com vistas em assegurar o direito humano à alimentação adequada e dá outras providências. Diário Oficial da União. 2006 set 15.

[14] Marques CF, Roberto NLB, Gonçalves HS, Bernardes AG. O que significa o Desmonte? Desmonte Do Que e Para Quem? Psicologia: Ciência e Profissão. 2019;39.

[15] Santarelli M, David G, Burity V, Rocha N. Informe Dhana 2019: autoritarismo, negação de direitos e fome. Brasília: FIAN; 2019.

[16] Silva W, Barbosa E. Austeridade e neoliberalismo no Brasil pós-golpe. Revista Sítio Novo. 2020;4(3):11.

[17] Vasconcelos FDAGD, Machado ML, Medeiros MATD, Neves JA, Recine E, Pasquim EMU, et al. Public policies of food and nutrition in Brazil: From Lula to Temer. Revista de Nutrição. 2019;32.

[18] Castro IRR. A extinção do Conselho Nacional de Segurança Alimentar e Nutricional e a agenda de alimentação e nutrição. Cadernos de Saúde Pública. 2019;35.

[19] Malta M. Human rights and political crisis in Brazil: Public health impacts and challenges. Global Public Health. 2018:1-8.

[20] Souza LEPFd, Barros RDD, Barreto ML, Katikireddi SV, Hone TV, Paes de Sousa R, et al. The potential impact of austerity on attainment of the Sustainable Development Goals in Brazil. BMJ Global Health. 2019;4.

[21] Arretche M. Federalismo e políticas sociais no Brasil: problemas de coordenação e autonomia. São Paulo em Perspectiva. 2004;18(2):17-26.
[22] Mattos RA, Baptista TWF. Caminhos para análise das políticas de saúde. Porto Alegre: Rede Unida; 2015.

[23] Tinôco I, Bezerra B, Lucio P, Barbosa L. Characterization of Rainfall Patterns in the Semiarid Brazil. Rio de Janeiro: UFRJ; 2018.

[24] UNDP. Human Development Atlas in Brazil Becerra: United Nations Development Programme 2010 [Internet]. 2103 [acesso em 2013 jun 15]. Disponível em: http:/ / atlasbrasil.org.br/2013/en.

[25] Atlas.ti scientific software development $\mathrm{GmbH}$. Qualitative data analysis. Version 7.5. 4. ed. Berlin: Atlas.ti; 2015.

[26] Ball SJ. What is policy? Texts, trajectories and toolboxes. Discourse Studies in the Cultural Politics of Education. 1993;13:8.

[27] Stuckler D, Reeves A, Loopstra R, Karanikolos M, McKee M. Austerity and health: the impact in the UK and Europe. European Journal of Public Health. 2017;27(suppl_4):18-21.

[28] Borges FT, Fernández LAL, Campos GWDS. Políticas de austeridade fiscal: tentativa de desmantelamento do Sistema Nacional de Salud da Espanha e resistência cidadã. Saúde e Sociedade. 2018;27:715-28.

[29] Boardman J. Dismantling the social safety net: social security reforms, disability and mental health conditions. BJPsych Bulletin. 2020;1-5.

[30] Mattei L. Les conséquences socio-économiques du coup d'État parlementaire au Brésil. Brésil(s) Sciences humaines et sociales. 2018(1).

[31] Nascimento RC. O papel do CONSEA na construção da política e do sistema nacional de segurança alimentar e nutricional [tese]. Rio de Janeiro: Universidade Federal Rural do Rio de Janeiro; 2012.

[32] Oliveira AR. Comida e aspectos simbólicos na perspectiva de políticas públicas para o cumprimento do Direito Humano à Alimentação Adequada e Saudável. Segur. Aliment. Nutr. 2020;27:e020025-e020025.

[33] Dos Santos Silva DA, Panelli-Martins BE. O processo de adesão municipal ao Sistema Nacional de Segurança Alimentar e Nutricional. Segur. Aliment. Nutr. 2020; 27:e020006-e020006. 
[34] Anderson MIP. Médicos pelo Brasil e as políticas de saúde para a Estratégia Saúde da Família de 1994 a 2019: caminhos e descaminhos da Atenção Primária no Brasil. Revista Brasileira de Medicina de Família e Comunidade. 2019;14(41):2180.

[35] Giovanella L, Bousquat A, Almeida PFD, Melo EA, Medina MG, Aquino R, et al. Médicos pelo Brasil: caminho para a privatização da atenção primária à saúde no Sistema Único de Saúde? Cadernos de Saúde Pública. 2019;35.

[36] Teixeira S, Silva R. Política de Assistência Social: entre o familismo e a desfamilização. Emancipação. 2020;20.

[37] Rocha JC. Soberania e Segurança Alimentar e no Semiárido. In: Conti I, Schroeder O. Convivência com o Semiárido Brasileiro: Autonomia e Protagonismo Social. 1. Brasília: IABS; 2013. p. 125-134.

[38] Castro MC, Massuda A, Almeida G, Menezes-Filho NA, Andrade MV, de Souza Noronha KVM, et al. Brazil's unified health system: the first 30 years and prospects for the future. The Lancet. 2019;394(14741547X).

[39] Paes-Sousa R, Schramm JMdA, Mendes LVP. Fiscal austerity and the health sector: the cost of adjustments. Ciência \& Saúde Coletiva. 2019;24:4375-4384.

[40] Machado CV, Silva GAE. Political struggles for a universal health system in Brazil: successes and limits in the reduction of inequalities. Globalization and Health. 2019;15(1):77.

[41] Ribeiro-Silva R, Pereira M, Campello T, Aragão E, Guimarães J, Ferreira A, et al. Implicações da pandemia COVID-19 para a segurança alimentar e nutricional no Brasil. Ciência \& Saúde Coletiva. 2020;25:3421-3430. 